\title{
Performance Analysis of IEEE 802.3 using IGRP and EIGRP Routing Protocols
}

\author{
Vishal Sharma \\ SBSCET, Ferozepur \\ Punjab, India
}

\author{
Rajneesh Narula \\ AIET, Faridkot \\ Punjab, India
}

\author{
Sumeer Khullar \\ AIET, Faridkot \\ Punjab, India
}

\begin{abstract}
With the growing need to distribute applications across multiple networks and the availability of high capacity, highperformance intermediate switching nodes and networks, an efficient routing mechanism has become the core requirement. This paper compares the performance of intra-domain routing protocols such as Enhanced Interior Gateway Protocol (EIGRP) and Interior Gateway Protocol (IGRP) of IEEE 802.3 LAN by evaluating various parameters including Network convergence time, End to End Delay, Delay Variation, Throughput, Utilization, Queuing Delay and IP Processing Delay. In addition to these metrics, we also compared the performance of video- and voice-data on the entire network under various constraints managed by routing protocols. Our simulation has been performed using the wellknown simulator OPNET.
\end{abstract}

\section{General Terms}

Routing Protocols, QoS parameters, IEEE 802.3 LAN

\section{Keywords}

IGRP, EIGRP, OPNET simulator

\section{INTRODUCTION}

Routing protocol is the key for the quality of modern communication network to propagate network topology information to the neighboring routers efficiently [1]. Efficient and scalable routing is one of the key challenges in the design and performance of a large scale intra-domain network $[1,4]$. Intra-domain routing protocols are basically dynamic routing protocols that are classified into two categories as distance vector routing protocol and link state routing protocol. The link state routing protocol and the distance vector routing protocol differ in that the former considers entire topology for routing decisions, whereas the latter considers only the information updated by the neighboring router [5] and [7-9]. A distance vector routing protocol works by advertising the information regarding the destination of the information together with the way to reach that destination. The implementations for the distance vector routing protocols incorporate the Bellman-Ford algorithm, in order for a router to update routing information of its neighbors within fixed intervals. A neighboring router then updates its distance vector value and subsequently, the updates are propagated to its neighbors. A simple configuration of the distance vector routing protocols makes them popular to be widely used. However, their routing mechanism makes them suitable only for small networks where the performance is not the main priority [13-14]. In Intra-domain networks, there are a number of parameters that evaluate the performance of the network such as End to End delay, the time taken by a packet to travel across the network from source to destination, Delay variation, the fluctuation of end-to-end delay from packet to the next packet, Throughput, the average rate of the packets successfully transmitted over a communication link [5-6] and [11-12]. Similarly, IP processing delay, the time consumed by a router for processing an IP packet, Utilization describes the load of the router's processor for processing IP packets. Similarly, the point-to-point queuing delay, the time consumed for the processing of IP queues. All these parameters significantly impact the performance of a routing protocol being used for any network to evaluate its performance.

Mainly, there are four routing protocols used such as RIP, OSPF, IGRP and EIGRP protocols. A metric hop count is used by RIP while routing. In comparison with other protocols, it is popular mainly due to the ease of use, administration and configuration. Starting with the source router, the maximum hop count of 15 nodes is allowed, otherwise the destination is considered to be unreachable [34]. In addition, it has a large support for classless inter-domain routing (CIDR) together with the multicast mechanism. OSPF is a link state routing protocol $[2,4]$ that allows routers to learn updates dynamically and send this updated information to every other node in the network. It supports classless interdomain routing and is considered very efficient as it uses Shortest Path First (SPF) algorithm [6-7]. IGRP is a cisco proprietary protocol and is better than RIP since it provides unequal-cost load sharing and an efficient update packet format. However, it is limited to cisco compliant products, in contrast to RIP which can be configd on any platform on which IP routing process is enabled $[10,16]$. Due to the limitations of RIP, IGRP comes into picture to overcome the problems. IGRP is a distance-vector routing protocol used within an autonomous system (AS). EIGRP [17] is an advanced distance-vector routing protocol that relies on features commonly associated with link-state protocols. OSPF's best traits, such as partial updates and neighbor discovery, are similarly put to use by EIGRP [4], [11], [15]. As we mentioned before, IGRP is a distance vector Interior Gateway Protocol (IGP). Distance vector routing protocols mathematically compare routes using some measurement of distance. This measurement is known as the distance vector. Routers using a distance vector protocol must send all or a portion of their routing table in a routing update message at regular intervals to each of their neighboring routers. As routing information distributes through the network, routers can identify new destinations as they are added to the network, learn of failures in the network, and calculate distances to all known destinations. We can also note that IGRP does not support VLSM where EIGRP does. VLSM (variable length subnet masking) allows you to subdivide a classful network into subnets. Also be aware that EIGRP and IGRP are compatible with each other [12]. Creation of EIGRP is to answer the increasing needs in networking and demands of diverse, large-scale internetworks. EIGRP transports the 
subnet mask information, which makes it a Classless routing protocol. EIGRP contains an important protocol called Diffusing update algorithm (DUAL) [2] that enables EIGRP routers to determine whether a path advertised by a neighbour is looped or loop-free, and allows a router running EIGRP to find alternate paths without waiting on updates from other routers. Therefore, EIGRP minimizes both the routing instability triggered by topology changes and the use of bandwidth and processing power in the router. Whenever, a change in the network happens, the routers with EIGRP sends out just the changes to the routing table. An automaticredistribution mechanism allows IGRP routes to be imported into EIGRP, and vice versa. Because the metrics for both protocols are directly translatable, they are easily comparable. IGRP and EIGRP path selection is based on Bandwidth/Delay metric. Using some EIGRP setting maximum bandwidth can be changed as required. The performance of the combination of IS-IS/RIP protocol shows better performance compared to EIGRP protocol in terms of throughput and end-to-end delay whereas, the network convergence of EIGRP protocol is better than IS-IS/RIP protocol [7]. The performance comparison between OSPF, RIP, IGRP and EIGRP protocols, EIGRP provides much better network performance than IGRP, RIP and OSPF during communication in simple as well as in bulky networks [12]. The delay variation is highest in case of OSPF protocol unlike the EIGRP- and RIP- protocols while the end to end delay is worst in case of RIP and slightly better in EIGRP than the OSPF [13].

In our study, we use to analyze the performance of intradomain routing protocols. In this paper, a detailed study aimed at the comparison of intra-domain routing protocols like IGRP (distance vector protocol) and EIGRP (hybrid protocol), containing the properties of both distance vector and link state routing protocols, have not been undertaken before altogether, is reported by evaluating the parameters of network traffic, IP processing delay, utilization, point-to-point throughput and point-to-point queuing delay, End to End delay, Delay variation for video and voice transmission. The paper is organized as Section I presents a star topology based framework incorporating intra-domain routing protocols, Section II provides simulation study followed by Section II reporting results and discussion of a simulative scenario implementing different routing protocols.

\section{SIMULATION SETUP}

This section presents our simulation setup to compare the performance of the intra-domain routing protocols. For our simulation setup, we have used the OPNET simulator that provides support for a large number of network protocols. It provides a high level user interface that is built on $\mathrm{C} / \mathrm{C}++$ code for supporting various routing protocols through a large number of library functions [37, 39]. The simulation model of IEEE 802.3 LAN network of area $10 \mathrm{~km}$ as shown in Fig 1 consist of two Ethernet gateways (ethernet4_slip8_gtwy) connected using PPP_DS3 links and four Ethernet Workstation name as video server, video client and voice workstations connected using PPP_DS1 links.

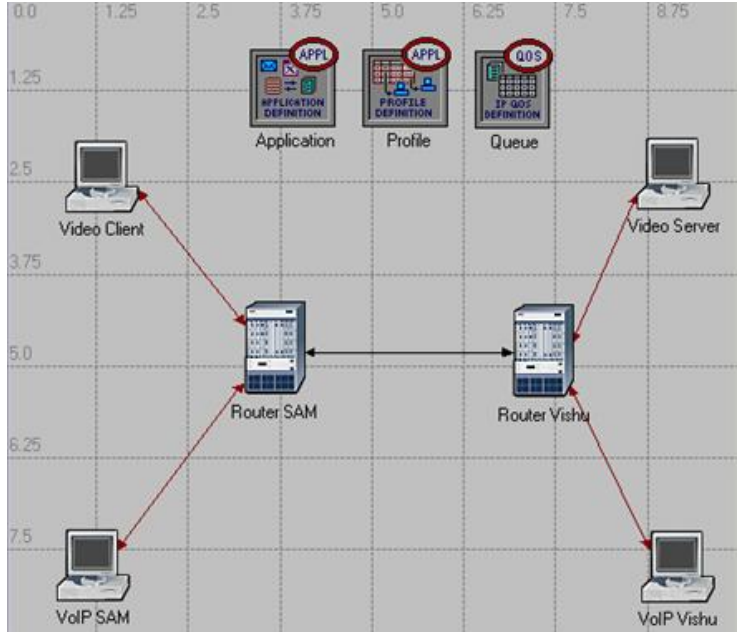

Fig 1: Simulation Framework of IEEE 802.3 LAN network for supporting video- and voice- data

An Application Definition Object and a Profile Definition Object named correspondingly as Application Config and Profile Config is added from the object palette into the workspace [18]. The Application Config allows generating different types of application traffic in conjunction with real time applications. The Application Definition Object is set to support video streaming and voice conferencing (PCM Quality). A Profile Definition Object defines the profiles within the defined application traffic of the Application Definition Objects. In the Profile Config, two profiles are created. One of the Profiles has the application support of video streaming and another one has voice conferencing of PCM quality support. One Video server is connected to Router Vishu that is set to the video streaming under the supported services of the video server. Two VoIP workstations are connected to each other by routers which are set to the VoIP under the supported services of the voice workstations. The routers are connected using PPP DS3 duplex link with each other. The other all workstations and video server are connected to routers using PPP_DS1 duplex link.

\section{RESULT \& DISCUSSION}

In this section, we evaluate results of IEEE 802.3 network investigated for simulation period of 15 minutes by means of OPNET simulator incorporating IGRP- and EIGRP- routing protocols by computing End to End delay, delay variation $(\mathrm{sec})$, point-to-point throughput (bits/sec), point-to-point queuing delay (seconds), traffic received (bits/sec), IP processing delay (seconds) for video and VoIP data traffics. Fig 2-3 shows that in case of end-to-end delay for the both data streams, the IEEE 802.3 network perfoms better when used with IGRP routing protocols as compare to EIGRP enabled network. Further, the graphs shows that the end-toend delay is more for video data stream as compare to VoIP traffic for simulation period of 15 sceonds. The network bears more packet delay variations in case of EIGRP as compare to IGRP in both cases of video and voice data streams and exhibits less variations in case of video traffic as depicted in Fig 4-5. 


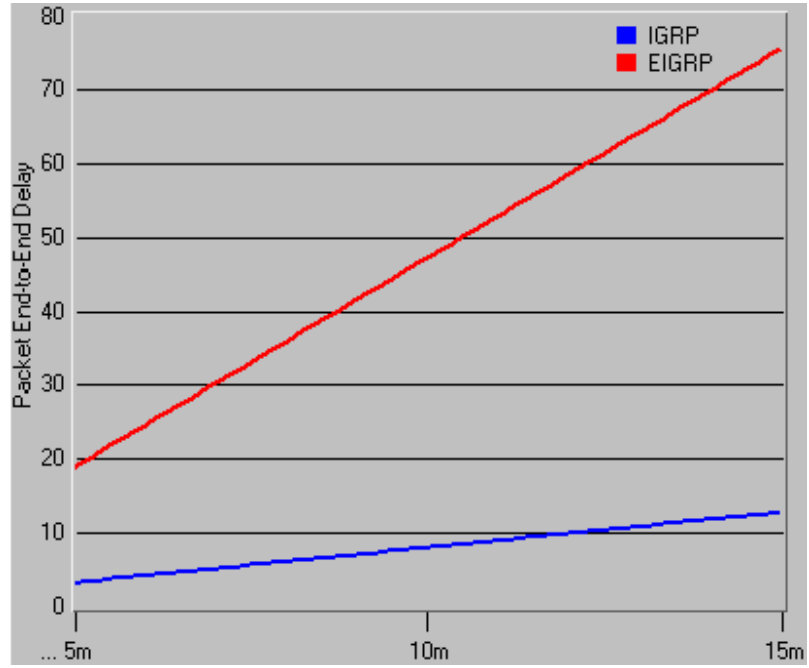

Fig 2: End to End delay in seconds using different Routing protocols for video transmission

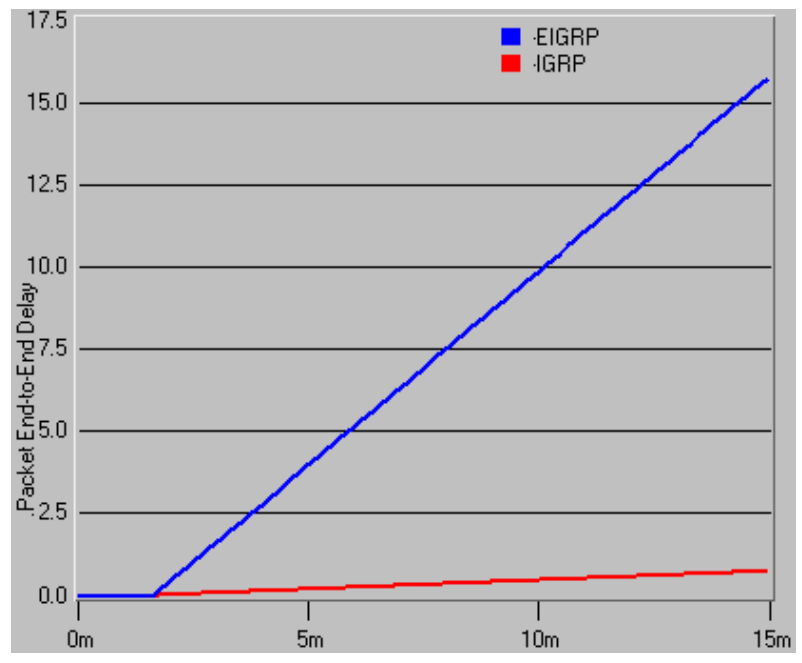

Fig 3: End to End delay in seconds using different Routing protocols for voice transmission

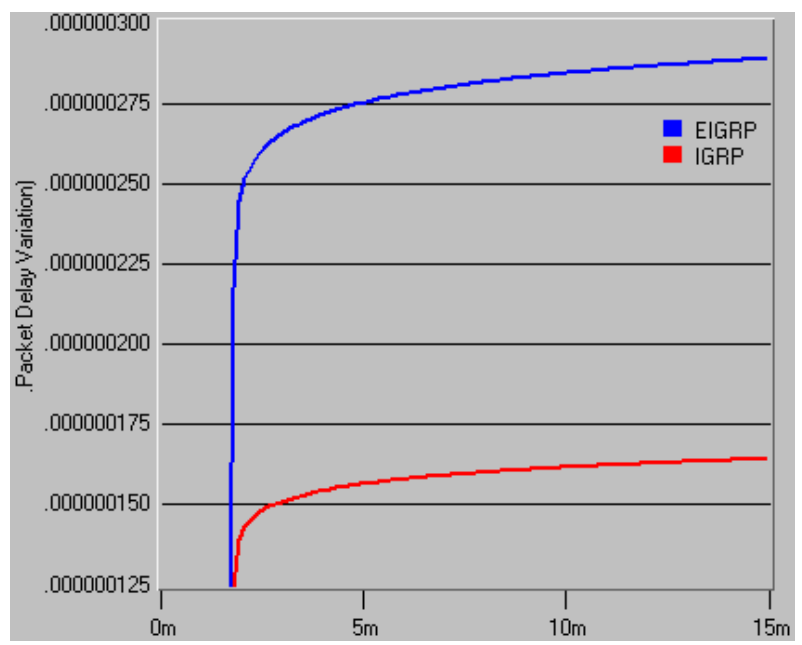

Fig 4: Packet delay Variation in seconds using different Routing protocols for video transmission

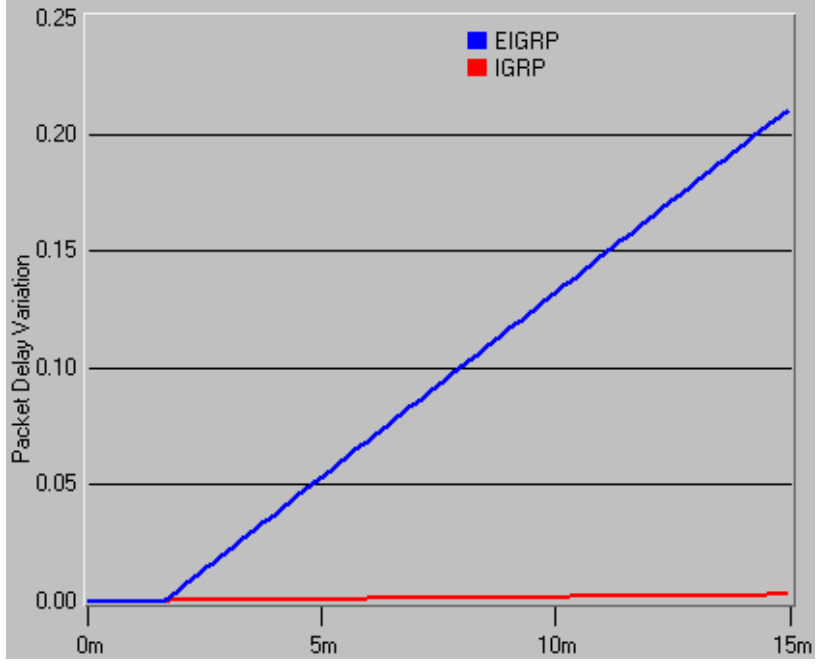

Fig 5: Packet delay Variation in seconds using different Routing protocols for voice transmission

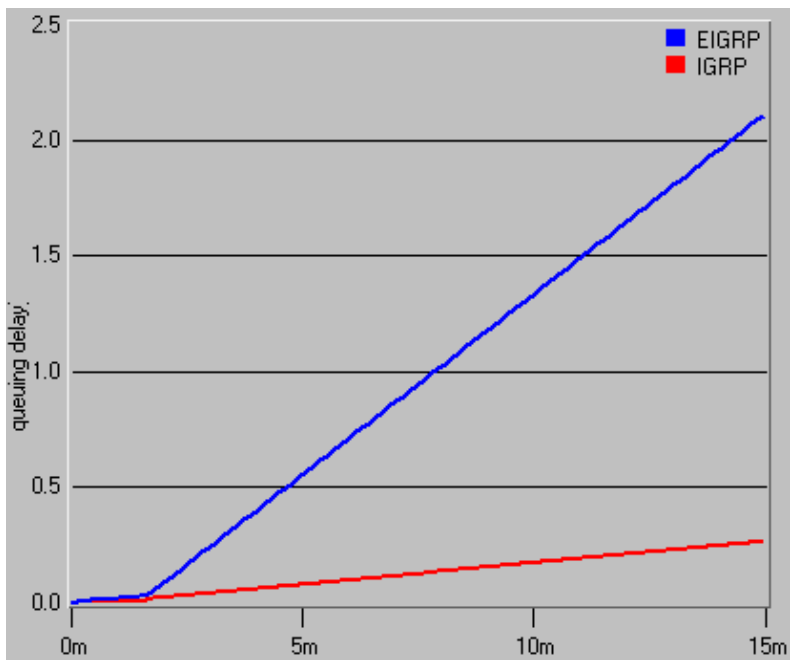

Fig 6: Point to Point (queuing delay) using different Routing protocols for video transmission

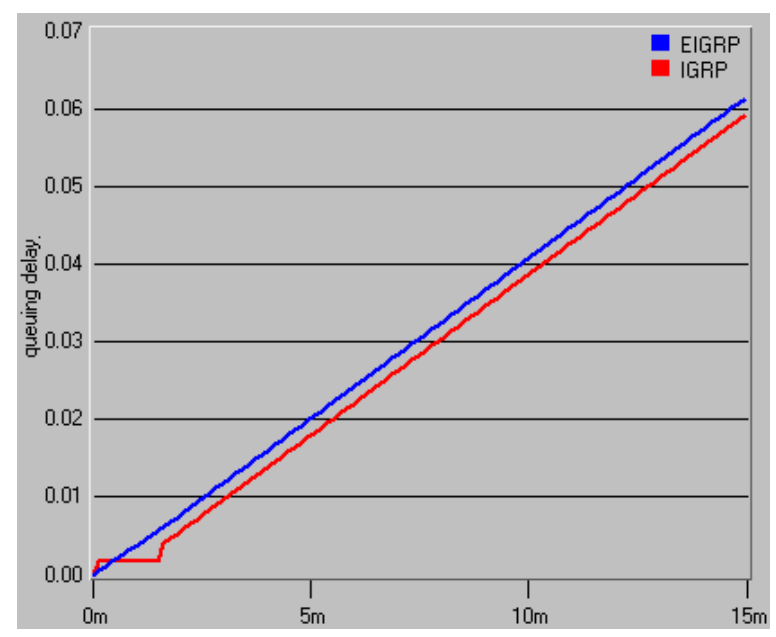

Fig 7: Point to Point (queuing delay) using different Routing protocols for voice transmission 


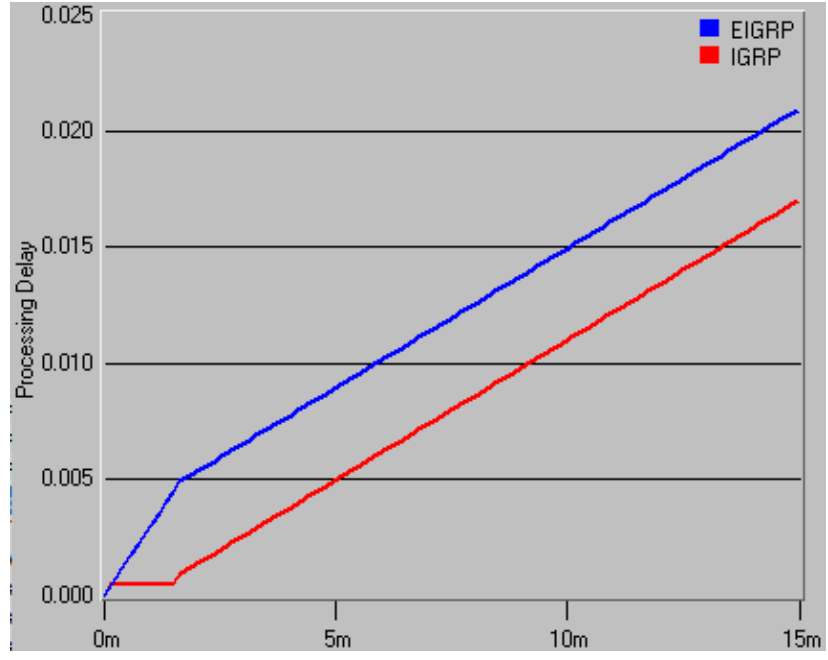

Fig 8: IP Processing Delay in sec using different Routing protocols for Router

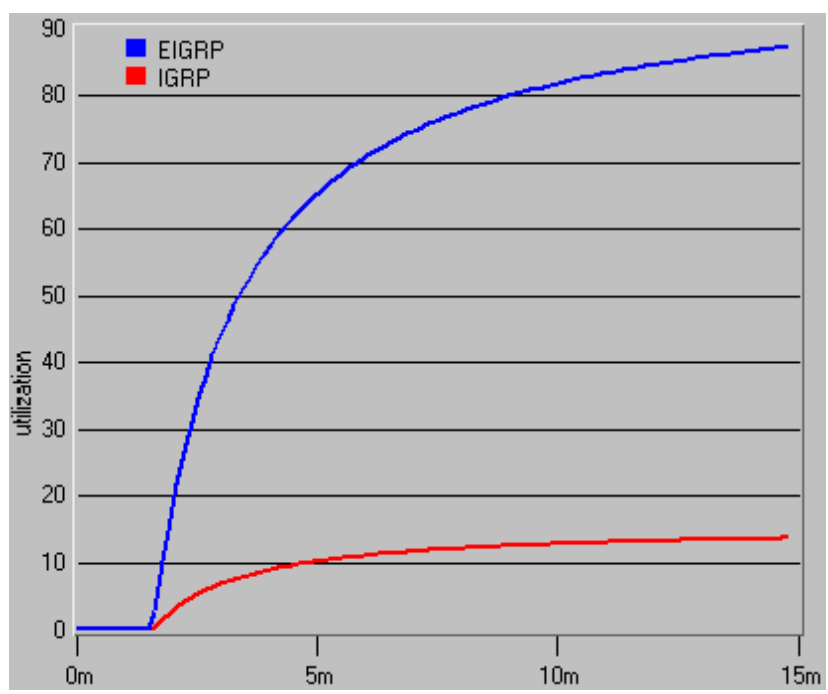

Fig 9: Point to Point (Utilization) using different Routing protocols for Video transmission

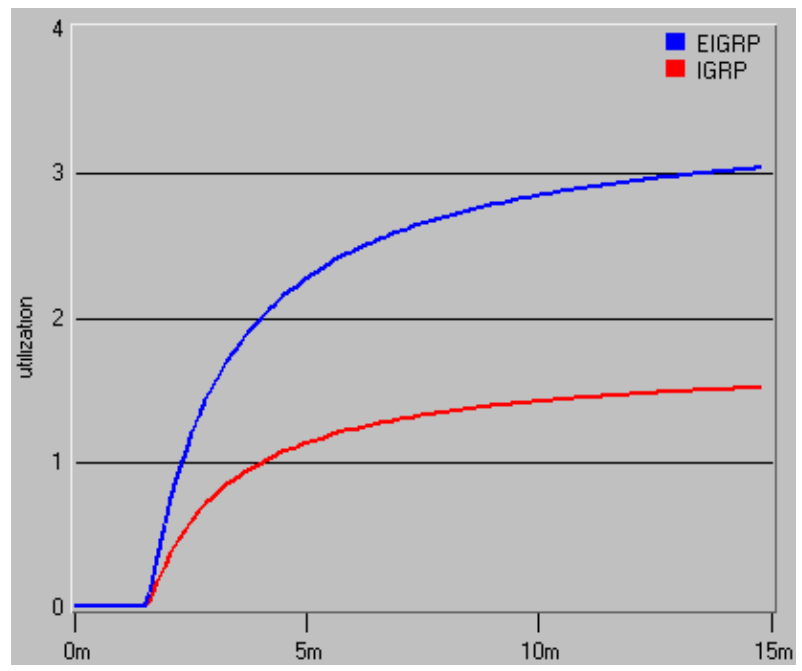

Fig 10: Point to Point (Utilization) using different Routing protocols for voice transmission

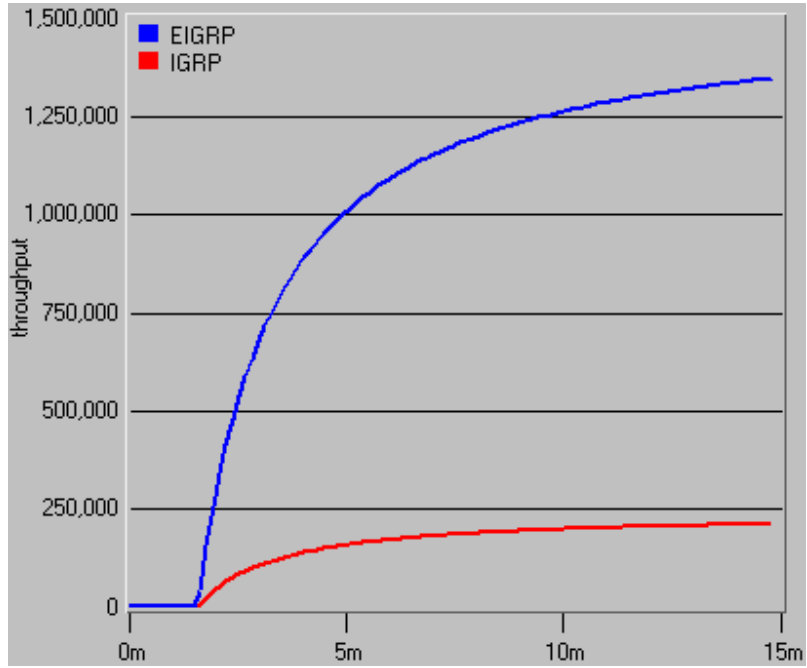

Fig 11: Point to Point (Throughput) using different Routing protocols for Video transmission

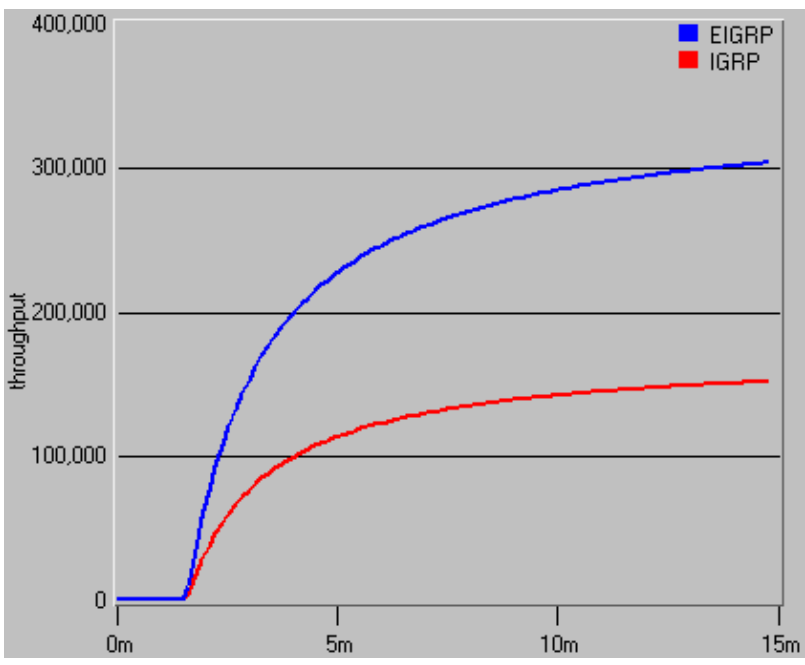

Fig 12: Point to Point (Throughput) using different Routing protocols for Voice transmission

Fig 6-7 shows that in veiw of point to point queuing delay for the both data streams, the IEEE 802.3 network perfoms better when used with IGRP routing protocols as compare to EIGRP enabled network which implies that the data packets have to wait for a longer average waiting time at routers that increase the probability of drop of data packets in case of EIGRP enabled networks. Further, the graphs shows that the point to point queuing delay is more for video data stream as compare to VoIP traffic. The IP processing delay for our network also evaluated as low by incoporating IGRP routing protocols as compare to EIGRP enabled network as shown in Fig 8. Furthermore, Figs 9-10 shows that the point to point utilization, a parameter related to the use of the channel disregard of throughput, of our simulative network is more with EIGRP routing protocols than that of IGRP scheme in both the cases of video- and voice- data stream which reveals that the routing devices running EIGRP require more processing power than that of IGRP routing protocols due to large overhead incurred with EIGRP routing protocol. Utilization parameter counts not only with the data bits but also with the transmission overhead, consists of preamble sequences, frame headers and acknowledge packets, which make use of the channel. Otherwise, the throughput would not 
be only associated to the efficiency of the protocol but also to retransmissions resultant from quality of the channel. In a simplistic approach, channel efficiency can be equal to channel utilization. The point to point throughput, a key parameter to determine the rate at which total data packets are successfully delivered and received through the channel in the network, of our simulative network is more with EIGRP routing protocols than that of IGRP scheme in both the cases of video- and voice- data stream as depicted in Fig 11-12.

\section{CONCLUSION}

This article compares the performance of two Intra-domain routing protocols EIGRP and IGRP for IEEE 802.3 network of $10 \mathrm{Km}$ by using OPNET simulator and performed simulations to examine the behaviour of these routing protocols using different parameters such as end to end delay, delay variation, point-to-point utilization, point-to-point throughput, point-to-point queuing delay and IP processing delay for video- and voice- based data traffic. Our results reveal out that IGRP routing protocol enabled networks performs better than that of EIGRP. The EIGRP protocol behaves well in terms of point-to-point throughput but by considering other important network parameters, calculated in this work, used to evaluate the efficiency of a network, it is recommended IGRP routing protocol to be used to deploy in small to medium sized network

\section{REFERENCES}

[1] Tanenbaum, Andrews, 2003. "Computer Networks", Pearson Education.

[2] Mohammad Nazrul Islam, 2010. Simulation Based EIGRP over OSPF Performance Analysis, Master Thesis in Electrical Engineering Emphasis on Telecommunications, Thesis no: 4983.

[3] Rick Graziani and Allan Jonson, 2008. Routing protocols and concepts: CCNA exploration companion guide, Pearson Education, London.

[4] Dong (Don) Xu, Ospf, 2011. EIGRP and RIP performance analysis based on Opnet, "Communication Networks", Springer.

[5] IKram Ud Din, Saeed Mahfooz and Muhammad Adnan, 2010. Analysis of the Routing protocols in Real Time Transmission, Global Journal of Computer Science and Technology, 10 (2010),18-22,

[6] Mohammad Nazrul Islam, 2010. Simulation Based EIGRP over OSPF Performance Analysis, Master Thesis in Electrical Engineering Emphasis on Telecommunications, Thesis no: 4983
[7] Esuendale Shewandagn Lemma, Syed Athar Hussain, Wendwossen Worku Anjelo, 2010. Performance Comparison of EIGRP/ IS-IS and OSPF/ IS-IS (Thesis)", Master Degree, Electrical Department, Blekinge Institute of Technology.

[8] Franck Le, Geoffrey G. Xie, Hui Zhang, 2010.Theory and new primitives for safely connecting routing protocol instances, 40 (2010), Issue 4, 219-230.

[9] R. M. Pethe and S. R .Burnase, 2010. Technical Era Language of the Networking- EIGRP", International Journal of Engineering Science and Technology, NCICT Special Issue, 1-5.

[10] Mohamad Yehia, S. Aziz and Hussein Elsayed, 2011. Analysis of IGP Routing Protocols for Real Time Applications, 3 (2011), Issue 6, 11-17.

[11] Wu, Bing, "Simulation Based Performance Analyses on RIP, EIGRP and OSPF Using OPNET",http://digitalcommons.uncfsu.edu/macsc_wp/1 $1,2011,1-24$.

[12] Anja Feldmann, Jennifer Rexford, 2011. IP Network Configuration for Intra-domain Traffic Engineering" 15 (2011), Issue 5, 46-57.

[13] Zhou Haijun, Pan Jin, Shen Pubing 2003. Cost adaptive OSPF" Proceedings of Fifth International Conference on Computational Intelligence and Multimedia Applications ICCIMA 2003, 55-60,

[14] Kisten, S. and Ping-Tsai Chung. 2003 Analysis and experimentation on dynamic routing protocols: EIGRP and OSPF, International Conference on Internet ComputingIC'03, 2 (2003), 591-594.

[15] Thorenoor, S.G. 2010. Dynamic Routing Protocol Implementation Decision between EIGRP, OSPF and RIP Based on Technical Background Using OPNET Modeller, Proceedings of the Second International Conference on Computer and Network Technology (ICCNT 2010), 191196.

[16] Yee, J.R. 2006. On the International routing protocol enhanced interior gateway routing protocols: is it optimal? International Transactions in Operational Research, 13 (2006), Issue 3, 177-94.

[17] Al-Saud K.A., Tahir H.M., El-Zoghabi A.A., Saleh, M. 2008. Performance evaluation of secured versus nonsecured EIGRP routing protocol" Proceedings of the 2008 International Conference on Security \& Management (SAM 2008), 292-7.

[18] OPNET Documents, http://www.opnet.com 